\title{
Scatterometry reference standards to improve tool matching and traceability in lithographical nanomanufacturing
}

Emil Agocs ${ }^{\mathrm{a}}$, Bernd Bodermann ${ }^{\mathrm{a}}$, Sven Burger ${ }^{\mathrm{b}}$, Gaoliang Dai ${ }^{\mathrm{a}}$, Johannes Endres ${ }^{\mathrm{a}}$, Poul-Erik Hansen $^{c}$, , Lars Nielsen ${ }^{c}$ Morten Hannibal Madsen ${ }^{c}$ Sebastian Heidenreich ${ }^{a}$, Michael Krumrey ${ }^{\mathrm{a}}$, Bernd Loechel $^{\mathrm{d}}$, Juergen Probst ${ }^{\mathrm{d}}$, Frank Scholze ${ }^{\mathrm{a}}$, Victor Soltwisch ${ }^{\mathrm{a}}$, Matthias Wurm ${ }^{\mathrm{a}}$ ${ }^{a}$ Physikalisch-Technische Bundesanstalt, Bundesallee 100, 38116 Braunschweig and Abbestraße 212, 10587 Berlin; ${ }^{b} \mathrm{JCMwave}$ GmbH, Bolivarallee 22, D-14050 Berlin, Germany; ${ }^{\mathrm{c} D a n s k}$ Fundamental Metrologi, Matematiktorvet 307, DK-2800 Kongens Lyngby, Denmark; ${ }^{\mathrm{d} H e l m h o l t z-}$ Zentrum Berlin für Materialien und Energie GmbH, Albert-Einstein-Str. 15, D-12489 Berlin, Germany

\begin{abstract}
High quality scatterometry standard samples have been developed to improve the tool matching between different scatterometry methods and tools as well as with high resolution microscopic methods such as scanning electron microscopy or atomic force microscopy and to support traceable and absolute scatterometric critical dimension metrology in lithographic nanomanufacturing. First samples based on one dimensional $\mathrm{Si}$ or on $\mathrm{Si}_{3} \mathrm{~N}_{4}$ grating targets have been manufactured and characterized for this purpose. The etched gratings have periods down to $50 \mathrm{~nm}$ and contain areas of reduced density to enable AFM measurements for comparison. Each sample contains additionally at least one large area scatterometry target suitable for grazing incidence small angle X-ray scattering. We present the current design and the characterization of structure details and the grating quality based on AFM, optical, EUV and X-Ray scatterometry as well as spectroscopic ellipsometry measurements. The final traceable calibration of these standards is currently performed by applying and combining different scatterometric as well as imaging calibration methods. We present first calibration results and discuss the final design and the aimed specifications of the standard samples to face the tough requirements for future technology nodes in lithography.
\end{abstract}

Keywords: Scatterometry, OCD, ellipsometry, CD metrology, reference standard, traceability, tool matching

\section{INTRODUCTION}

Scatterometry, in the semiconductor industry often referred to as optical CD (OCD) metrology, is a generic term for a number of different important optical techniques to support the lithographic nanomanufacturing process in semiconductor industry. Scatterometric techniques are generally very sensitive to many relevant physical and dimensional features including the critical dimensions (CD, typically the feature width) of the nanosized structures to be manufactured and as optical methods they are fast, non-destructive and practically contamination-free. However, today scatterometry is usually not applied for absolute CD measurements and quality control. The main reason for this is caused by the lack of tool matching between scatterometers and CD-SEMs (scanning electron microscopes for CDMetrology), which are typically used as reference tools for CD metrology. In measurement comparisons scatterometers typically show an excellent linearity to CD-SEM tools. In many cases however, systematic offsets between both systems of the order of several $\mathrm{nm}$ up to few $10 \mathrm{~nm}$ [1-3] are observed. These systematic deviations may be connected both to the applied measurement methods and tools, to necessary approximations in the modeling and data analysis and to imperfections and limitations of the target structures. Although these systematic offsets may also be at least partly attributed to the CD-SEM measurements or simply to inconsistencies in the definition of the measurands, for the implementation of scatterometry as absolute and traceable metrology it is necessary to identify, characterize and eliminate possible causes for systematic measurement errors and to evaluate thoroughly a complete measurement uncertainty estimation to achieve reliable scatterometric measurement results [4-6]. Recently, we have investigated and quantified several of these possible systematic error sources [7-9]. However, this is a quite elaborate task, which could be made easier and manageable for practical instrustrial applications with the availability of suitable calibrated scatterometry reference standards.

*bernd.bodermann@ptb.de; phone +49 531 592-4222; fax +49 531 592-4264;

http://www.ptb.de/cms/en/ptb/fachabteilungen/abt4/fb-42/ag-423.html 
Today, several CD standards based either on structured photomasks [10,11] or wafers [12-14] are available to support the metrology for lithographical nanomanufacturing. However, none of them is designed and suitable to test state-of-theart OCD tools, since they either contain only single calibrated structures $[10,12,14]$, the grating targets are too small for current tools [14] or the structures are quite large $(C D \gg 100 \mathrm{~nm})$ and only mask based [10, 11]. To overcome this shortcoming, within a joint research project (JRP) [15] we recently have developed, characterized and calibrated scatterometry standard samples to support the CD metrology in semiconductor industry, in particular for wafer processing including lithography. Furthermore it was aimed to enable tool validation for different type of OCD tools and to support the equalisation and matching of the various measurement techniques (OCD, SEM and AFM) used mainly for CD metrology in the semiconductor industry.

Two different standard samples, based either on $\mathrm{Si}$ or on $\mathrm{Si}_{3} \mathrm{~N}_{4}$, have been developed. We have characterized the structure quality on these samples using both high resolution microscopy such as Atomic Force Microscopy (AFM) and SEM, and different scatterometry methods such as optical, EUV as well as X-Ray scatterometry and spectroscopic ellipsometry. For the calibration a combined analysis of DUV and EUV scatterometry, spectroscopic ellipsometry or Mueller polarimetry and GISAXS measurement data supported by AFM and CD-SEM results will be applied using Bayes algorithms [16-18].

Here, we report on the status of these developments and discuss the final design and aimed specifications of these standard samples and of possible future extensions.

\section{SCATTEROMETRY REFERENCE STANDARD SAMPLES}

The design of the scatterometry reference standards had to take into account different boundary conditions and requirements. So they should be applicable for different type of instruments both of the project partners and of course especially for end users in industry, should cover state of the art industry requirements and current lithography technologies and be extendable to future technology steps. Additionally a principal suitability for AFM and SEM characterization was desirable as well. And finally the manufacturability and availability of high quality manufacturing processes was of course another important condition. We have developed two scatterometry standards, a Si- and a resist mimicking dielectric standard based on $\mathrm{Si}_{3} \mathrm{~N}_{4}$. The different type of standard samples and materials are chosen to cover different metrological applications like resist metrology and inspection of the fabricated wafer, so that various metrological requirements in semiconductor industry are gathered.

A well-controlled state of the art manufacturing is of key-importance in view of high reproducibility. The reference standard samples were manufactured by electron beam lithography. The substrates used were silicon wafers for the silicon gratings and silicon wafers with a deposited $100 \mathrm{~nm}$ silicon nitride layer for the dielectric gratings.

Suitable processes for the manufacturing of both $\mathrm{Si}$ and $\mathrm{Si}_{3} \mathrm{~N}_{4}$ versions of the reference standards have been identified and optimised. The substrates were spin coated with positive electron beam resist ZEP520A, with thicknesses down to $30 \mathrm{~nm}$ for the smallest CD of $25 \mathrm{~nm}$. For the electron beam exposure, a Vistec EBPG5000+ES e-beam writer was used, which operates with an electron acceleration voltage of $100 \mathrm{kV}$. The final step involves reactive ion etching of the substrate, while the developed resist acts as etch mask. The etching gases used were $\mathrm{SF}_{6}$ and $\mathrm{C}_{4} \mathrm{~F}_{8}$ for the silicon gratings and $\mathrm{CHF}_{3}$ for the dielectric gratings. Finally, the remaining resist layer was removed with an oxygen plasma. The electron beam writer addresses typically main field sizes of $250 \mu \mathrm{m}$ x $250 \mu \mathrm{m}$. Further distribution of the design over the whole sample size is done by scanning the sample itself via a laser interferometrically controlled stage. Although the individual fields are periodically aligned by an automatic adjustment of the beam deflection on designated markers on the sample holder table during the exposure, small drifts of the sample or holder system due to slight variations in temperature may lead to minor stitching errors between adjacent fields. Usually, these stitching errors are too small to have an impact on the design or to be measured e.g. by scanning electron microscopy. Interestingly, even the periodicity of sub-fields of $4.3 \mu \mathrm{m}$ in the e-beam writing process are visible in the GISAXS measurement at the PTB.

So far the produced reference standards cover a range of grating periods between $50 \mathrm{~nm}$ and $250 \mathrm{~nm}$ and nominal CD values between $25 \mathrm{~nm}$ and $100 \mathrm{~nm}$. The structure height was adapted for different grating periods to ensure the best manufacturing quality.

For the process development, testing and validation and to test the applicability of different metrology tools in a first step we used a design as shown in figure 1 . The large cross shaped grating was chosen to enable GISAXS measurements with the plane of incidence along and perpendicular to the grating lines. The scatterometric measurement areas for smaller 
spot size instruments are indicated by unique alignment marks (not shown in figure 1). Additionally there are several small areas (size $5 \mu \mathrm{m} \mathrm{X} 5 \mu \mathrm{m}$ ) with a reduced line density for AFM testing.

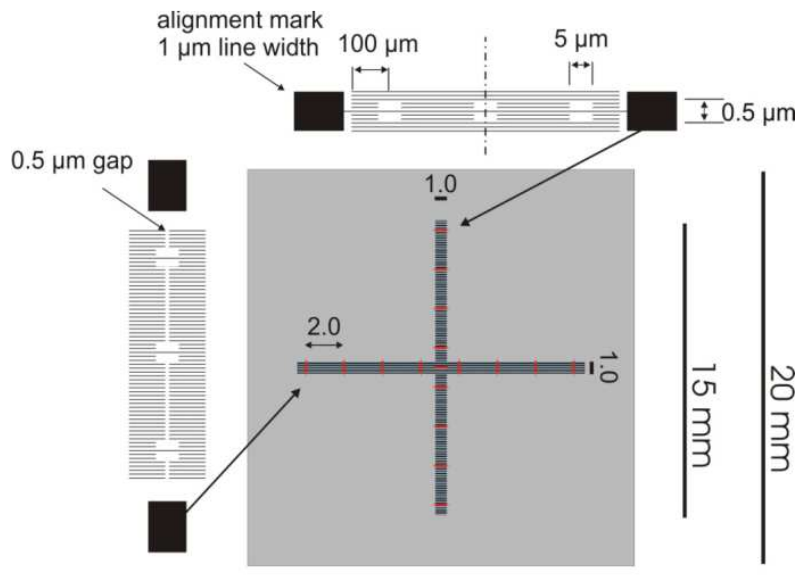

test fields for AFM

free standing line in $0.5 \mu \mathrm{m}$ by $5 \mu \mathrm{m}$

centre of patterned area and $100 \mu \mathrm{m}$ from edge

$2 \mathrm{~mm}$ distance along the grating bars and in centre of cross

Figure 1. Design of the first test samples to test and validate the manufacturing processes as well as the applicability of different metrology tools. The large cross shaped grating was chosen to enable GISAXS measurements with the plane of incidence along and perpendicular to the grating lines. The red lines mark areas of locally lowered line-to-space ration to enable AFM reference measurements even for small periods below $100 \mathrm{~nm}$.

\section{CHARACTERIZATION OF THE STANDARD SAMPLES}

To enable finally a calibration of the most important structure parameters CD, side wall angle (SWA), structure height and mean pitch (period) especially for the scatterometric measurements it is essential to gather as much a-priori information as possible about the structures to be measured to support unique and accurate measurement results. Therefore, and in order to validate the high quality of the fabricated grating samples, in a first step we thoroughly investigated detailed geometry features as well as the optical material parameters of the line structures.

To determine reliable optical material parameters, the complex refractive indices, we applied goniometric reflectometry, spectroscopic ellipsometry and spectroscopic Mueller polarimetry measurements in sufficiently large etched and not etched areas. For this purpose we used PTB's homebuilt 'DUV' scatterometer [3] and a commercial spectroscopic ellipsometer/Mueller polarimeter (SENTECH SENresearch 850SE). These optical parameters are typically strongly correlated with potential layer compositions and heights as well as etch depth or the corresponding structure heights. Therefore we additionally applied X-ray reflectometry and AFM measurements to characterize the layer structures and etch depth. With this approach we determined suitable mean values as a-priori information and (partly) good starting values for the final calibration procedure (see below) for both sample types:

- Si-samples: n\&k for the silicon and the (inevitable) silicon oxide layer; thickness of oxide layer; etch depth

- $\mathrm{Si}_{3} \mathrm{~N}_{4}$-samples: $\mathrm{n} \& \mathrm{k}$ for the silicon and the $\mathrm{Si}_{3} \mathrm{~N}_{4}$ layer; thickness of and etch depth in the $\mathrm{Si}_{3} \mathrm{~N}_{4}$ layer

Additionally we have applied different high resolution microscopy tools, namely low voltage SEM, cross section SEM and PTB's 3D AFM [19] to characterize further important structure details such as line edge roughness, edge angles or etch profile details such as corner rounding. 

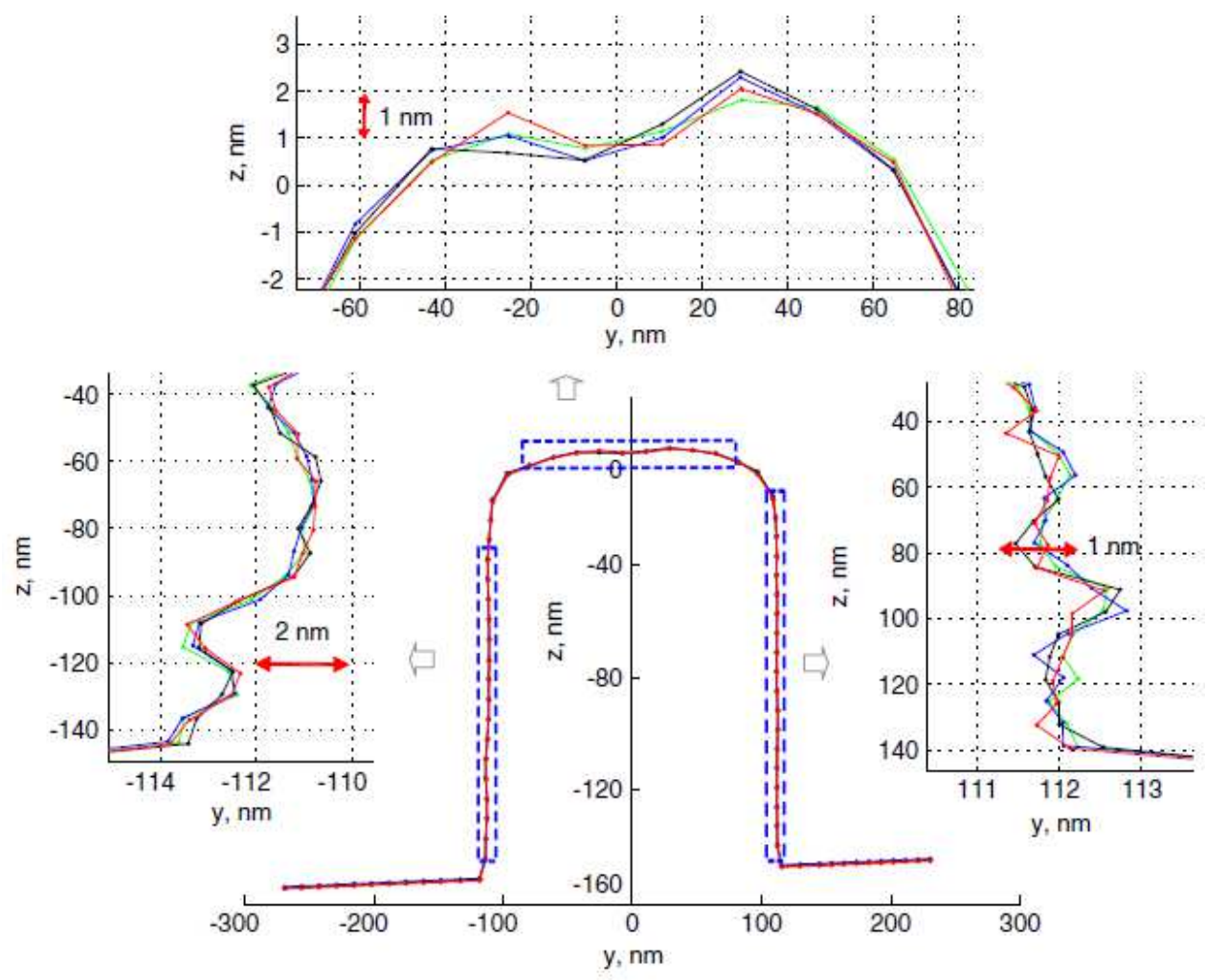

Figure 2: Measurement example of AFM line edge and line widths roughness measurements on test samples of the first iteration stage (with worse structure quality than the final samples).

Besides the structure height AFM measurements are utilized to characterize line edge roughness, line edge angles and profiles. Figure 2 shows an example of a Si line structure measured by our 3D-AFM. It demonstrates the excellent reproducibility of the measurements both in the vertical and in the lateral direction.

Figure 3, 4 and 5 show SEM measurement examples of the final standard structures for both materials, Si as well as $\mathrm{Si}_{3} \mathrm{~N}_{4}$. Measurements have been done using top down images (fig. 3) as well as cross section images (fig 4, 5), the latter of course only on identically manufactured test samples, because cross section imaging is a destructive method.

The top down SEM images shows rather smooth line edges. Applying PTB's edge detection algorithms [20], these top down images are analyzed to derive the local line edge roughness and to some extend also local line edge angles.

Even though SEM cross section images give only very indirect information about the samples to be characterized, since only identically manufactured samples are destructively measured instead of the samples of interest, they nevertheless give quite valuable information about the basic cross section geometry of the grating structures, provided, that the manufacturing process is stable and highly reproducible.

For the manufactured $\mathrm{Si}_{3} \mathrm{~N}_{4}$ gratings the cross section images (fig. 4) indicate, that the edges are not perfect, but show edge angles, which are significantly lower than the intended $90^{\circ}$. However, the observed top and bottom corner rounding is relatively small.

For the Si gratings (c. f. fig. 5) the observed edge angles are much steeper (about nearly $90^{\circ}$ ). However, as confirmed by the scattering data below, the edges show a significant bottom corner rounding. In fact the whole grooves in the bottom are rather curved with almost constant radius across its full width. The top corners of the lines on the other hand are much better defined. This is easily understood from the fabrication process, as the grooves are etched after resist development with the top area of the lines covered by the resist acting as the etch mask and thus protected. After etching, the resist is stripped by an oxygen plasma treatment which does not further etch the sample. This treatment, however, oxidizes the surface and causes the rather large oxide thickness of around $6 \mathrm{~nm}$. It should be noted that this oxide is not a stoichiometric $\mathrm{SiO}_{2}$ and probably also not homogeneous from the silicon interface to the surface. 


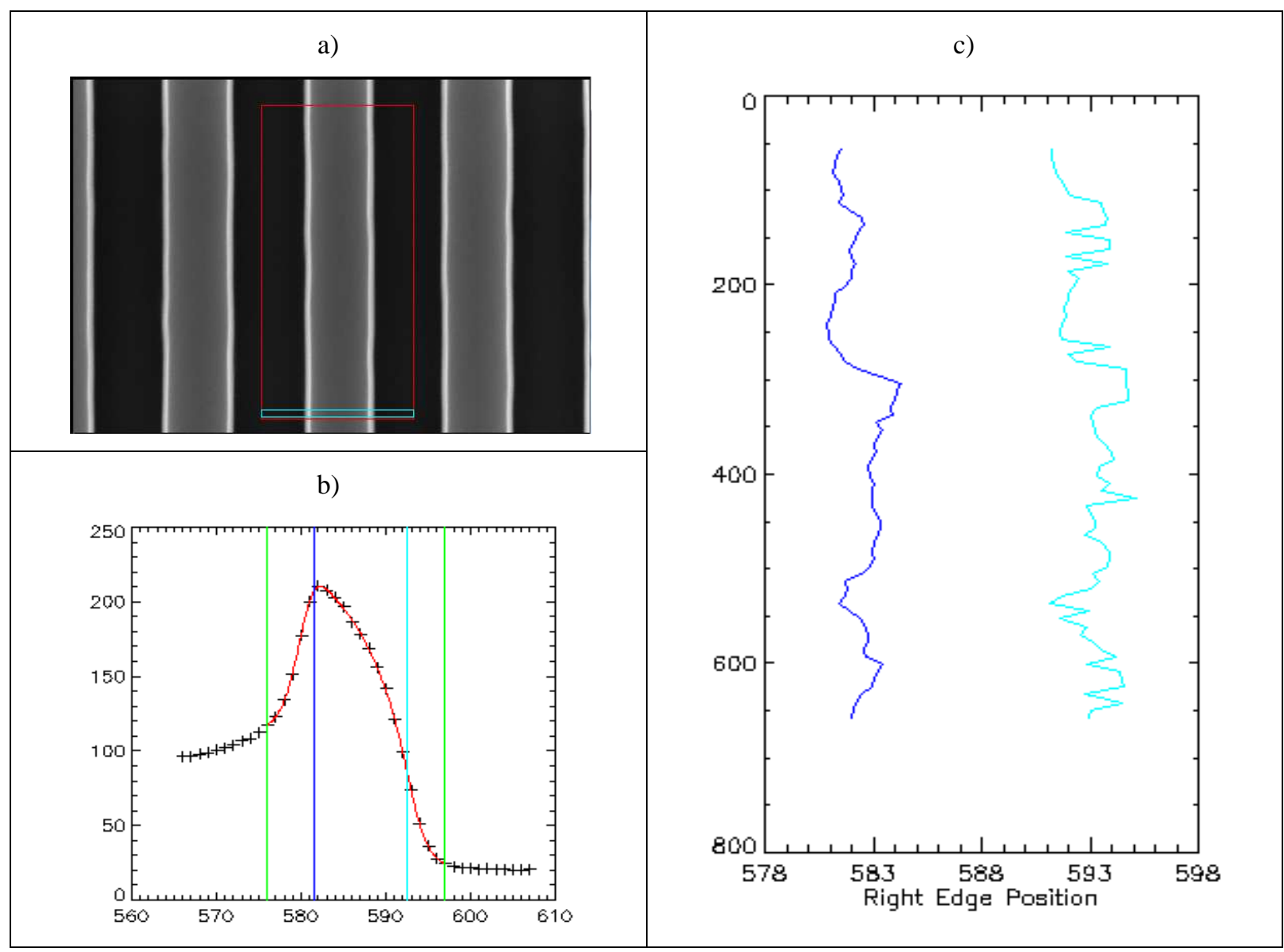

Figure 3: a) SEM top down image; b) analysis of edge positions applying PTB's BDF-edge detection algorithm; c) top (blue) and bottom (cyan) edge position versus scanning position to derive local line edge roughness parameters

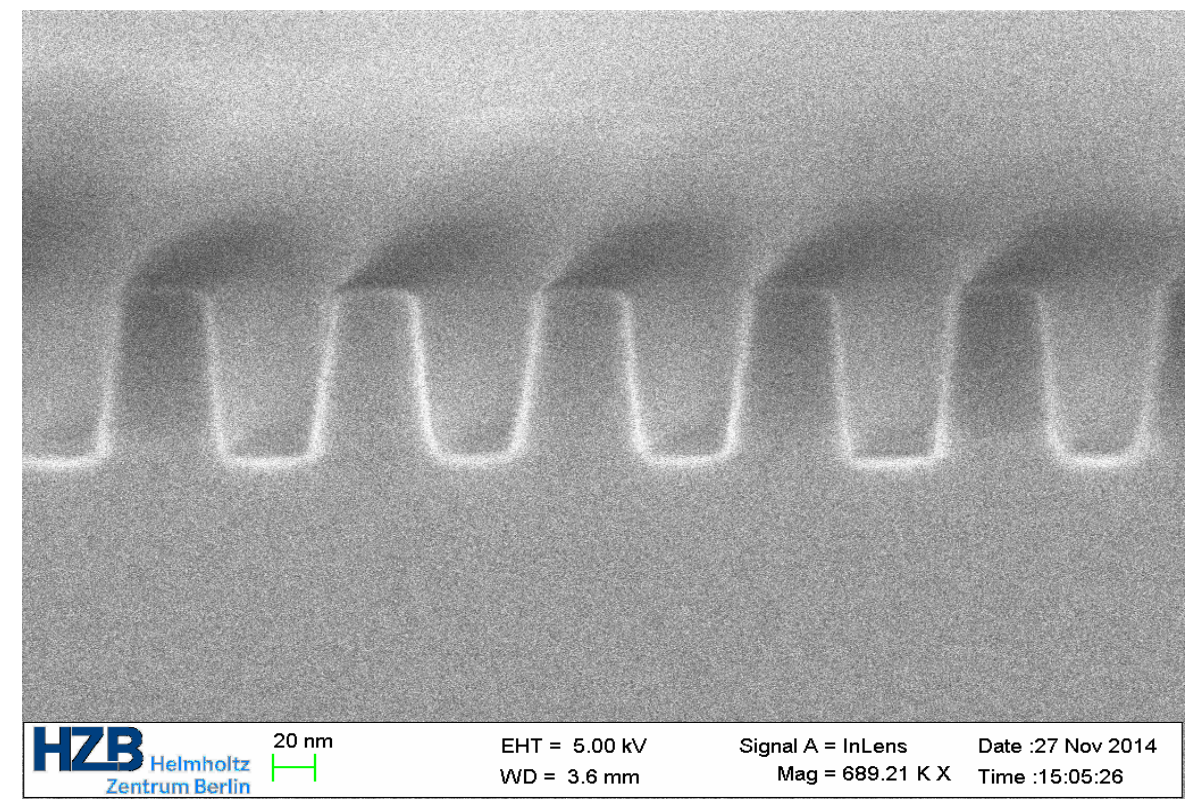

Figure 4: SEM cross section image of a $\mathrm{Si}_{3} \mathrm{~N}_{4}$ sample with a grating period of $100 \mathrm{~nm}$ and a nominal $\mathrm{CD}$ of $50 \mathrm{~nm}$. 
The microscopic characterization has been supplemented and validated by the scatterometric measurements in the DUV and X-ray spectral range using EUV-SAS and GISAXS, as shown in section 5.

Here in particular the GISAXS measurements due to the very short wavelength have proven to be very sensitive to structure details such as line edge as well as surface roughness. In fact due to the GISAXS measurement geometry these measurements are very sensitive and useful to measure as well long range line inhomogeneities such as stitching or substitching effects of the e-beam writing process [21].

\section{STRUCTURE MODEL AND DATA ANALYSIS}

As already mentioned above, for the final measurements and calibrations of the grating structures besides CD-SEM and CD-AFM high resolution microcopy several different approaches of 'photon based scatterometry' from the x-ray (GISAXS) to the visible and near infrared spectral range (ellipsometry / Mueller polarimetry) are applied.

To avoid as far as possible any systematic measurement deviations it is of utmost importance that the measurement data of all methods are analyzed as far as possible with the same geometry and layer model and with the same data analysis methods. Therefore, all photon based measurements including the x-ray and EUV data is modeled and analyzed using the finite element based Maxwell solver JCMsuite [22, 23]. This FEM solver allows to model arbitrary structures and shows typically a very fast convergence, so that it is very well suited for the optimization process, which is required to derive the structure parameters from the scatterometric measurements.

For all results presented in this manuscript we have apply a two stage optimization: in a first step we use a global algorithm such as Particle Swarm or Differential Evolution to find a unique solution close to the absolute minimum of the multi-dimensional error function. In a second step we apply a local gradient based optimization in the vicinity of the global optimization result to refine the reconstruction result.

From the cross section SEM images (c. f. fig. 5) we have derived a reasonable geometry model for this optimization procedure. As shown in figure 5 (right) we use for all measurements a trapezoidal line cross section with top and bottom corner rounding as a suitable and sufficiently realistic structure profile description.

For the silicon samples we add a homogenous oxide layer on the Si-substrate as well as on the top surface and the edges of the silicon line structures.

For the $\mathrm{Si}_{3} \mathrm{~N}_{4}$ samples no such oxide layer is required. However, here we allow for an etch depth, which might differ from the height of the $\mathrm{Si}_{3} \mathrm{~N}_{4}$ layer to take into account a possible under- or over-etching of this nitride layer.
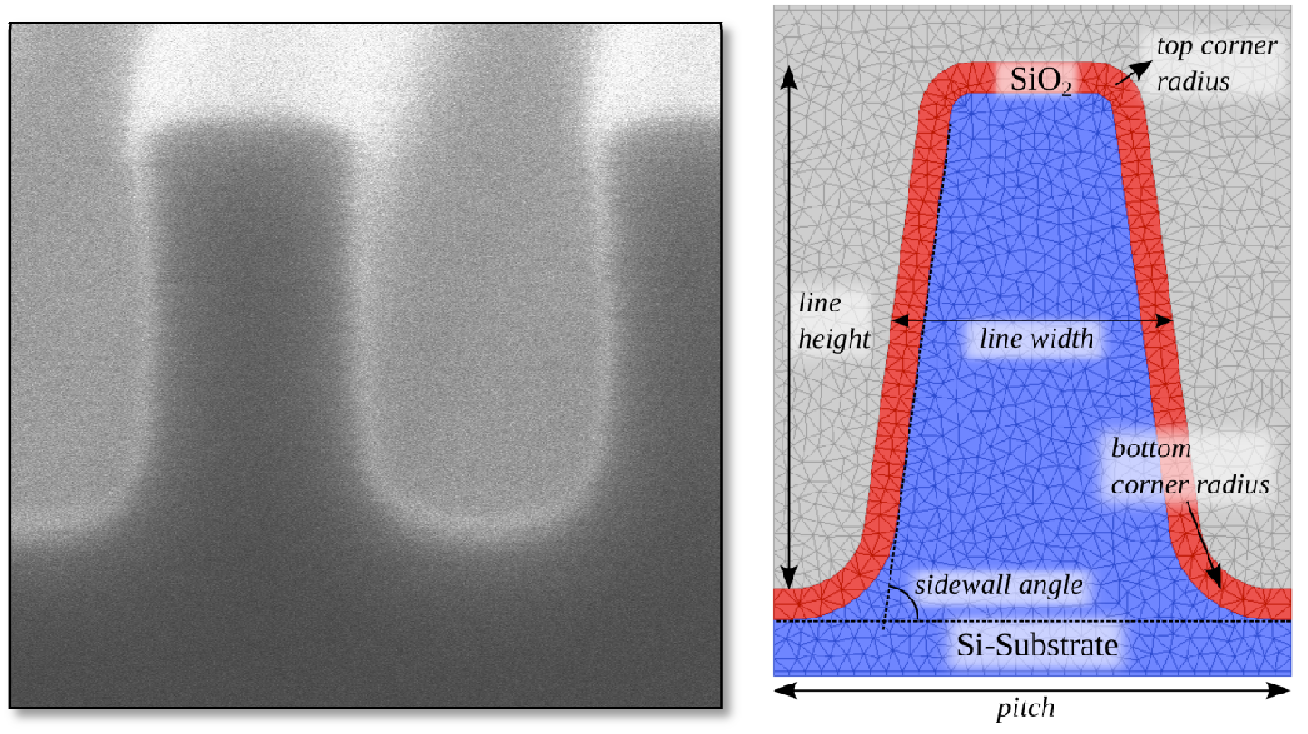

Figure 5. Left: cross section SEM image of a Si line structure, right: scheme of the geometrical model used for the data evaluation. The six parameters are indicated. For the model, we assumed a homogeneous thickness of the oxide, which results from oxygen plasma cleaning for resist stripping, at all surfaces. 


\section{MEASUREMENTS OF THE STANDARD SAMPLES}

For the final measurements and calibration of the scatterometry standard samples, besides the above mentioned microscopy methods CD-AFM and CD-SEM four different photon based methods and five different measurement systems are used:

- Spectroscopic Ellipsometry / Mueller polarimetry measurements are provided either by a home-build FourierMueller polarimeter operated in the VIS and NIR spectral range, developed recently by DFM [24], or by a versatile commercial DUV to NIR system (SENTECH SENresearch $850 \mathrm{SE}$ )

- Goniometric scatterometry measurements at an operation wavelength of $266 \mathrm{~nm}$ are done by PTB's home-build DUV-scatterometer $[25,26]$

- Small angle EUV scatterometry (EUV SAS, c. f. fig. 7) are performed with a unique EUV polarization sensitive reflectometer and scatterometer recently developed and set up at PTB at the electron storage ring BESSY II in Berlin [21]

- At another beam line of BESSY II another setup is applied for GISAXS measurements [21, 27]

We have measured all manufactured reference standard samples with all these different set-ups and methods and in a first step each measurement has been analyzed individually applying the model and approach described in the previous section.

Figure 6 illustrates the GISAXS measurement scheme and shows a measurement example of a Si standard sample compared with a best fit result of the FEM-optimization. This result documents the generally excellent fit quality.
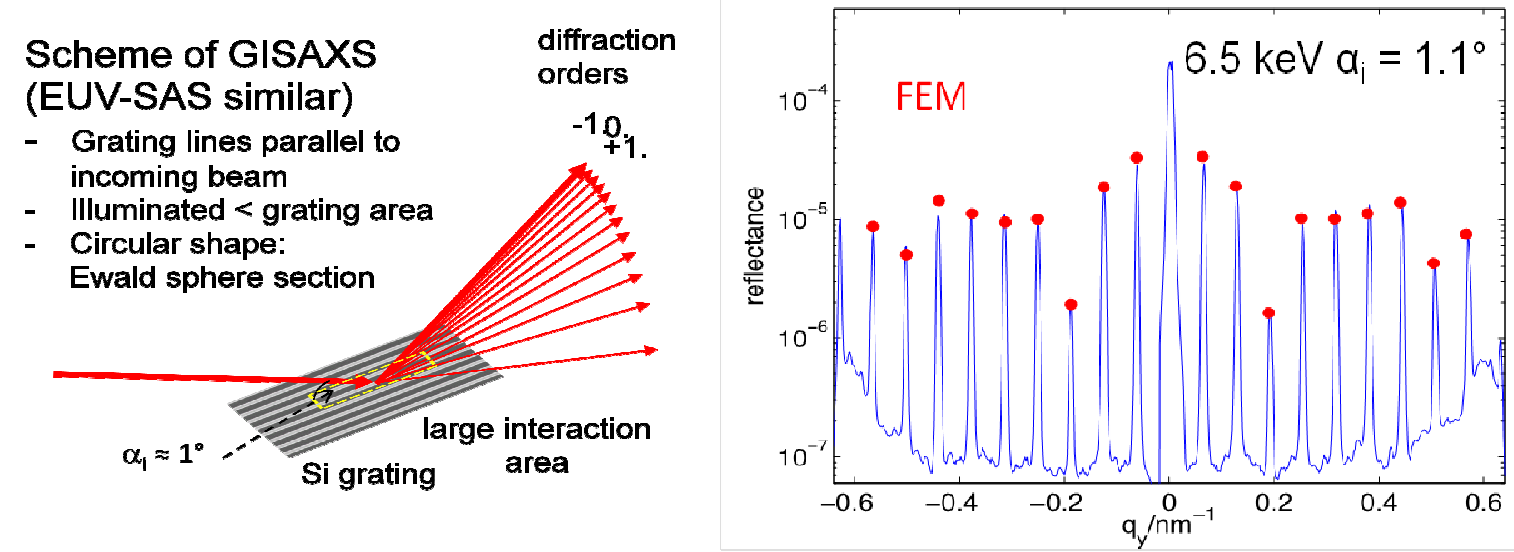

Figure 6: left: scheme of the GISAXS measurements at the PTB labs at BESSY II, right: comparison of FEM reconstruction (red squares) and diffraction efficiencies measured by GISAXS (blue line) of the grating sample with a pitch of $100 \mathrm{~nm}$. The angle of incidence is $88.9^{\circ}$; the indicated angle relies to the sample surface

Nearly comparable good results are obtained as well for the EUV-SAS measurements, as is shown in figure 7. Additionally shown is here a photo of the new and quite versatile EUV scatterometer and reflectometer system. Here, the measurement scheme is very similar to the GISAXS measurement. However, due to the much shorter wavelength the angle of incidence can be chosen significantly smaller, leading to strongly released requirements in the scatterometry target size in the direction of the plane of incidence.

Figure 8a shows the measurement scheme for the goniometric DUV scatterometer. Since the grating samples under test are in the sub-wavelength regime, usually only the zeroth diffraction order, i. e. the specular reflected beam is measurable in the far field. To extract nevertheless sufficient information about the structures we measured the samples in four different measurement geometries with the grating lines oriented within or perpendicular to the plane of incidence and illuminating the sample either with s- or p- polarized light. Figure 8 (bottom middle and right) shows measurement examples for two different Si gratings with periods of $100 \mathrm{~nm}(8 \mathrm{~b})$ and $50 \mathrm{~nm}(8 \mathrm{c})$, respectively. The comparison of the measured reflectance curves (dots) with the corresponding best fit optimization results (blue line) again shows an excellent agreement. The resulting structure geometries and sizes are shown as well and qualitatively compared with 

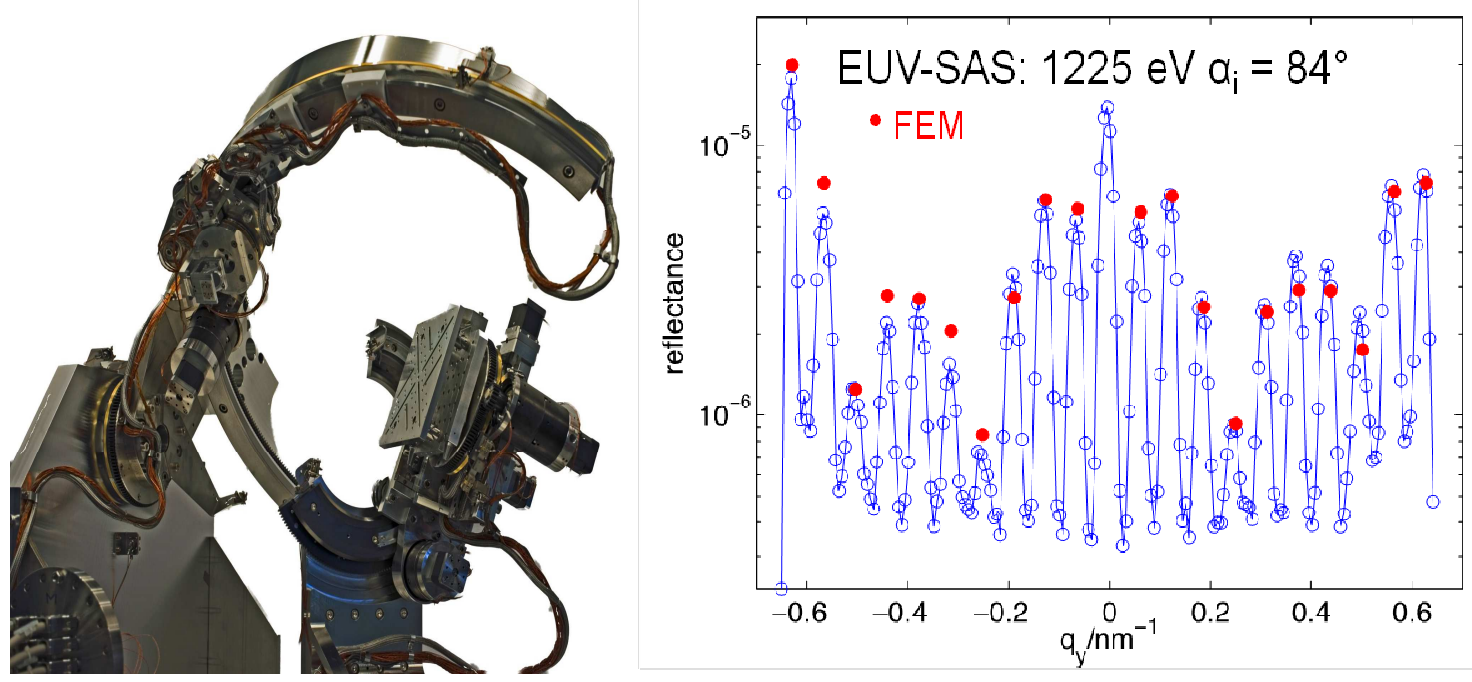

Figure 7: left: photo of the new EUV reflectometer/scatterometer system at the PTB labs at BESSY II, right: comparison of FEM reconstruction (red squares) and diffraction efficiencies measured by EUV-SAS (blue circles) of the grating sample with a pitch of $100 \mathrm{~nm}$. The angle of incidence can be chosen significantly smaller $\left(\sim 85^{\circ}\right)$ than for the GISAXS system, resulting in a strongly reduced interaction area.

a)

a)

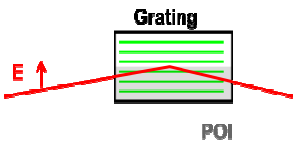

b)

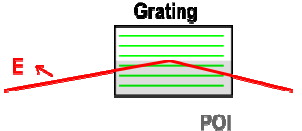

c)

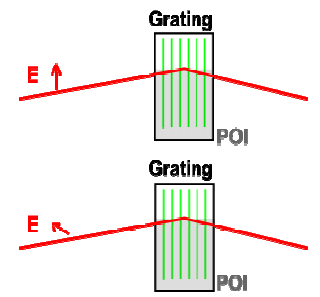

b)
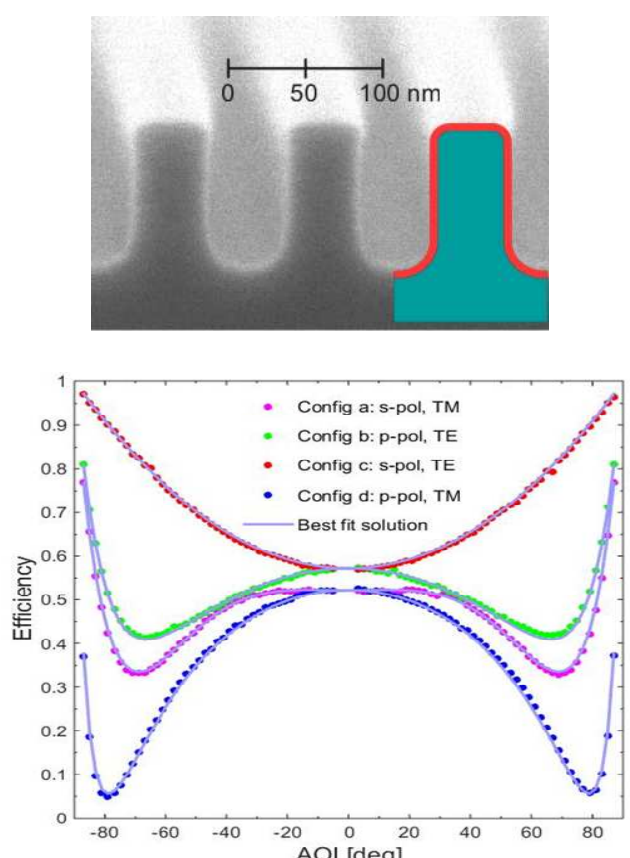

c)
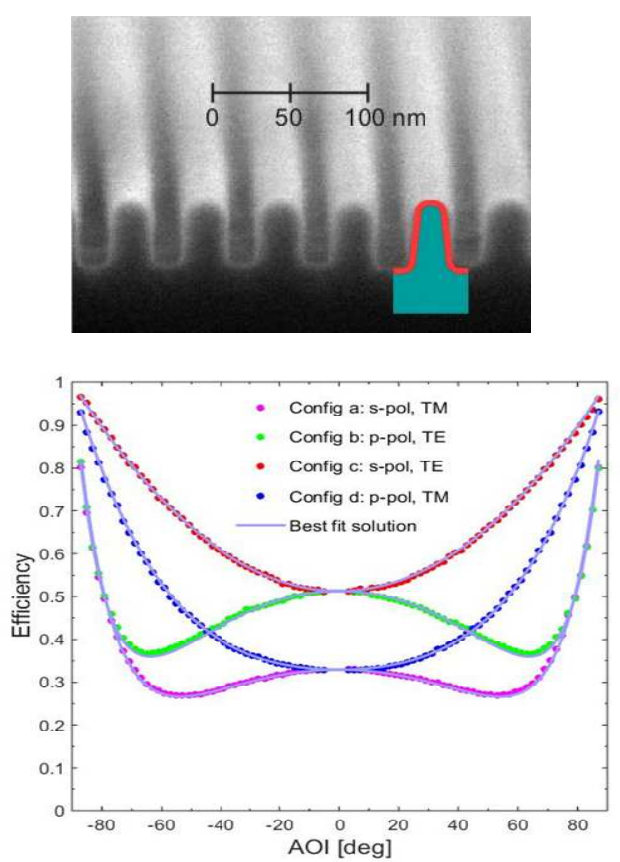

Figure 8: a) four different measurement geometries used for goniometric DUV scatterometry, b\&c) bottom: Comparison of FEM reconstruction (lines) and measured (dots) reflectance for grating sample with a period of $100 \mathrm{~nm}(\mathrm{~b})$ and $50 \mathrm{~nm}$ (c) and top: qualitative comparison of the structure geometries reconstructed from these measurements and the corresponding SEM cross section images.

corresponding SEM cross section images of nominal identical structures (8b, 8c, top). We would like to stress, that even tiny structure features such as the top and bottom corner rounding are obviously reconstructed very well, even if the size is in the deep sub-wavelength regime. Comparable good agreement is observed for the structure profiles obtained for the $\mathrm{X}$-ray scatterometry measurements, as compared with the SEM cross section images. 


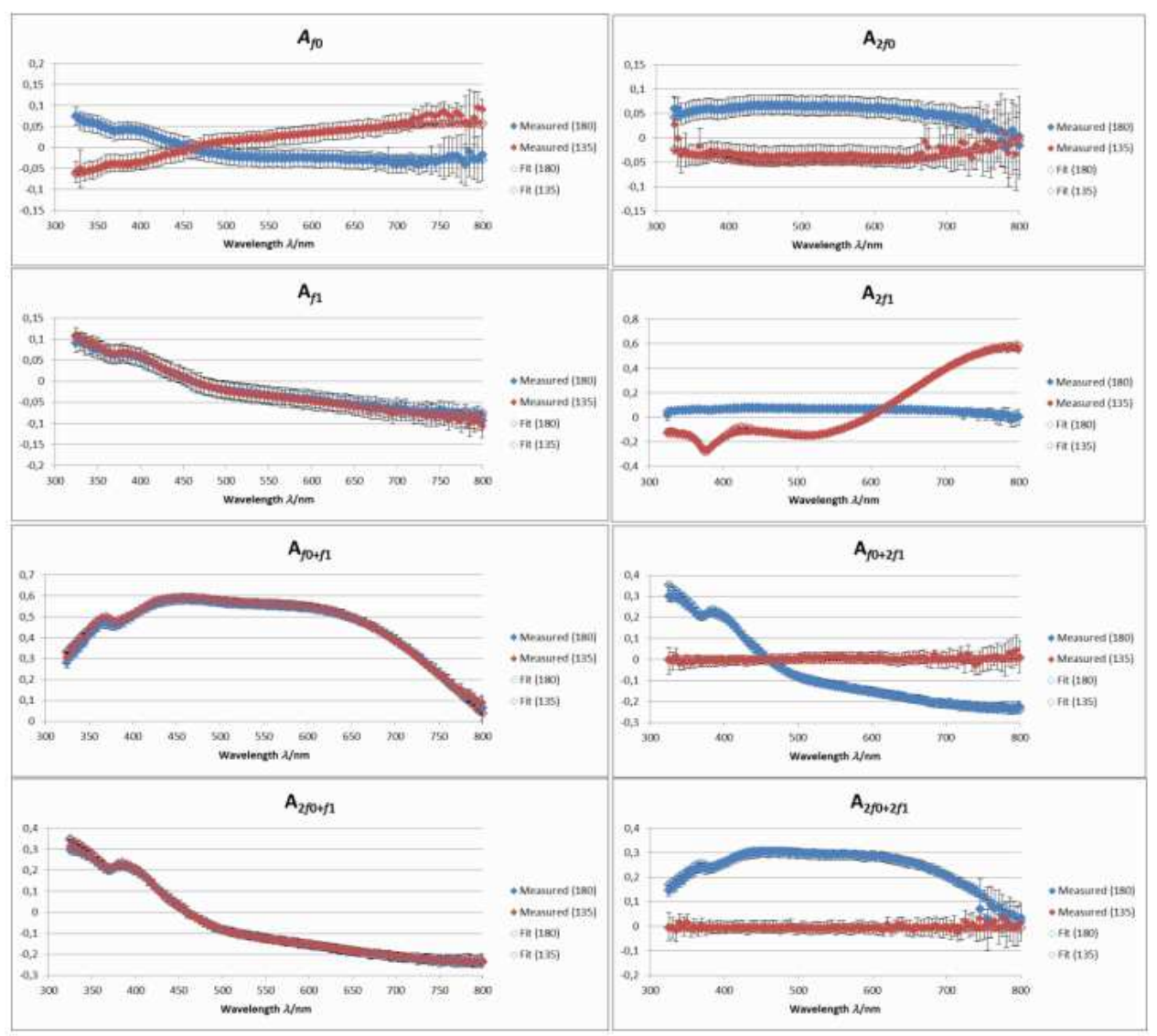

Figure 9: Mueller polarimetry measurement data for two different polarization state analyzer orientations $\left(180^{\circ}\right.$ and $\left.135^{\circ}\right)$ and best fit optimization results for a Si grating with a period of $100 \mathrm{~nm}$ and a nominal CD of $35 \mathrm{~nm}$ obtained with DFM's home build spectroscopic Mueller polarimeter. The matrix elements are given in terms of the corresponding Fourier coefficients.

Finally, figure 9 depicts a measurement example obtained with DFM's Mueller polarimeter. Here 8 of the 16 Mueller matrix elements are shown in terms of the corresponding Fourier coefficients, as obtained in the data analysis of this polarisation modulated measurement system. Measurements are shown for two different orientations of the polarization state analyzer with respect to the plane of incidence, and are compared with the corresponding best fit optimisation results. Again we observe a very good fit quality.

Table 1 shows results for the main structure parameters CD, height, side wall angle and corner roundings for three different Si grating targets obtained by individual evaluation of the described scatterometric measurements. The results for the obtained $\mathrm{CD}$ and height values are already in very good agreement $(\leq 2 \mathrm{~nm})$, in particular for the DUV and the Xray scatterometry measurements. For the oxide layer we observed some sensitivity issues for the X-ray scatterometry methods. The agreements of the results for the side wall angles and top as well as bottom corner rounding are reasonable, but not perfect. A reason for this might be some residual correlations between side wall angle and corner rounding.

Table 1: First individual measurements results on three different Si grating targets obtained by different scatterometric measurement methods and tools.

\begin{tabular}{|c|c|c|c|c|c|c|}
\hline & $\begin{array}{c}\mathrm{CD} \\
/ \mathrm{nm}\end{array}$ & $\begin{array}{c}\text { Height } \\
/ \mathrm{nm}\end{array}$ & Side Wall angle/ $/$ & \multicolumn{2}{|c|}{ Corner Radius } & $\begin{array}{c}\text { Oxide } \\
\text { Height } / \mathrm{nm}\end{array}$ \\
\cline { 5 - 7 } & & & Top $/ \mathrm{nm}$ & Bottom $/ \mathrm{nm}$ & . \\
\hline Si-target with nominal CD $=25 \mathrm{~nm}$, period $=50 \mathrm{~nm}$, height $=50 \mathrm{~nm}$ \\
\hline GISAXS & 25.1 & 48.2 & 87.7 & 4.2 & 13.8 & - \\
\hline
\end{tabular}




\begin{tabular}{|r|c|c|c|c|c|c|}
\hline DUV-Scatt. & 24.8 & 51.7 & 84.4 & 4.5 & 9.5 & 5.0 \\
\hline EUV-SAS & 23.3 & 48.9 & 88.6 & 6.4 & 11.6 & 4.7 \\
\hline Si-target with nominal CD $=55 \mathrm{~nm}$, period $=100 \mathrm{~nm}$, height $=100 \mathrm{~nm}$ \\
\hline GISAXS & 55.0 & 102.1 & 82.9 & 5.7 & 14.0 & - \\
\hline DUV-Scatt. & 53.4 & 101.2 & 90.0 & 8.0 & 20.5 & 5.3 \\
\hline EUV-SAS & 53.6 & 100.8 & 87.6 & 2.9 & 15.8 & 8.7 \\
\hline Si-target with nominal CD $=35 \mathrm{~nm}$, period $=100 \mathrm{~nm}$, height $=100 \mathrm{~nm}$ \\
\hline EUV-XRR & 31.5 & 104.2 & 89.3 & 3.9 & 25.3 & 5.8 \\
\hline DUV-Scatt. & 34.2 & 103.8 & 90 & 7.55 & 24.6 & 5.2 \\
\hline Mueller-Pol. & 37.0 & NA & 87.5 & 16.7 & 33.3 & 6.3 \\
\hline
\end{tabular}

\section{CONCLUSION AND OUTLOOK}

We have presented here the the current status of the development of high quality scatterometry reference standard samples. We have shown the development, characterization and first steps for the calibration of these samples. The manufacturing process and design for high quality samples has been developed. Characterization of the first manufactured test samples performed with a large variety of different high end tools confirms the good quality of the manufactured samples. Additionally first results of the calibration of Si-samples performed with different scatterometric tools and methods already show a good agreement.

Current measurement uncertainties for the different measurements for example for the $\mathrm{CD}$ values are estimated to be of the order of few $\mathrm{nm}$. However, to achieve even significantly smaller measurement uncertainty values for the final standard calibration a combined data analysis of all measurements including AFM, SEM and polarimetry data will be applied. The procedures and methods for this hybrid metrology approach based on Bayes algorithm have already been developed and tested $[17,18]$. With this approach we expect to reach measurement uncertainties $(\mathrm{k}=2)$ of below $1 \mathrm{~nm}$ for the $\mathrm{CD}$ and $0.3^{\circ}$ for the side wall angles.

First $\mathrm{Si}_{3} \mathrm{~N}_{4}$ samples have been manufactured as well and are currently characterized and calibrated in a similar way. The structure quality appears to be quite good as well, with even much reduced corner rounding, as compared with the silicon samples. The edges seem to be significantly less steep than the silicon line edges, but are assumed to be still appropriate for the intended purpose.

For the final version of the standard samples we have developed a modified design as shown in figure 10. As it was recognized, that for the GISAXS evaluation the test fields with the grating lines along the plane of incidence are much more appropriate and sufficient for the characterization of the grating structures, here we omitted the GISAXS field with grating lines perpendicular to the plane of incidence. Instead, to increase the practical use for state-of-the art OCD tools we added a matrix of scatterometry targets of $1 \mathrm{~mm}^{2}$ in size and with systematic variations in the grating period (currently $50 \mathrm{~nm}$ to $250 \mathrm{~nm}$ ) as well as the line-to-space-ratio (duty cycle, 0.9 to 1.1 ). Additionally we have added another column with a very low duty cycle of 0.25 to enable an improved and more direct comparison of OCD and AFM tools.

The grating periods and with it the structure dimensions may be scaled down to $22 \mathrm{~nm}$ and below to adjust to current and future technology nodes in semiconductor industry, provided the availability of suitable advanced e-beam writers and suitable resist. 
Both the final silicon and the dielectric reference standard samples will be available in the near future and the participating national metrology institutes will offer an adequate calibration service for these standards.

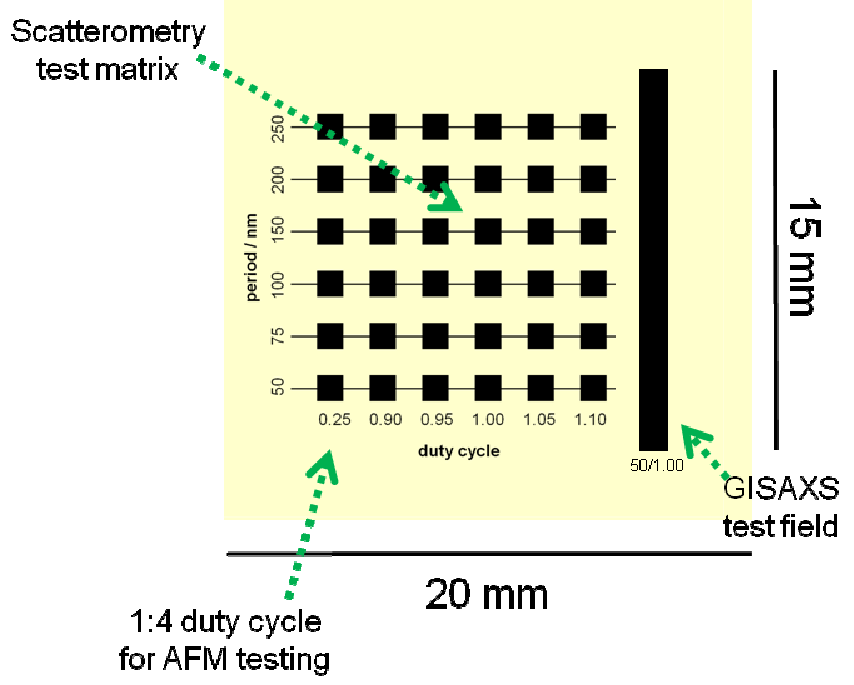

Figure 10: Draft of the final standard design containing only one GISAXS test field (plane of incidence along the grating lines) for GISAXS reference measurements and to test the process quality. Additionally a matrix of smaller $1 \mathrm{~mm}^{2}$ scatterometry targets is added to enhance the parameter space for scatterometer characterization. The low line-to-space-ratio in the left column enables a more direct comparison with AFM reference measurements even for small periods.

\section{ACKNOWLEDGEMENT}

This work is supported by several collaborators from industry, universities and research institutes. We would like to thank all collaborators, in particular ASML, Carl Zeiss AG and NIST for their support and for fruitful discussions. The project has been funded within the European Metrology Research Program (EMRP). The EMRP is jointly funded by the EMRP participating countries within EURAMET and the European Union. We thank the European commission and the EURAMET e. v. for financial support under the support code No 912/2009/EC.

\section{REFERENCES}

[1] P. Thony, D. Herisson, D. Henry, E. Severgnini, M. Vasconi: "Review Of CD Measurement and Scatterometry", AIP Conf. Proc. 683 (2003) 381-388

[2] C.J. Raymond: "Overview over scatterometry applications in high volume silicon manufacturing", AIP Conf. Proc. 788 (2005), 394-402

[3] M. Wurm, F. Pilarski, B. Bodermann: "A new flexible scatterometer for critical dimension metrology", Rev. Sci. Instrum. 81 (2010), 023701-1-023701-8

[4] Frank Scholze, Bernd Bodermann, Hermann Groß, Akiko Kato, Matthias Wurm: First steps towards traceability in scatterometry, Proc. SPIE 7985, (2011), 79850G.

[5] Heather J. Patrick, Thomas A. Germer, Progress toward traceable nanoscale optical critical dimension metrology for semiconductors, Proc. SPIE 6672, (2007), 66720L

[6] T. A. Germer, H. J. Patrick, R. M. Silver, B. Bunday: "Developing an Uncertainty Analysis for Optical Scatterometry", Proc. SPIE 7272 (2009), 72720T-1 - 72720T-11

[7] B. Bodermann, P.-E. Hansen, S. Burger, M.-A. Henn, H. Gross, M. Bär, F. Scholze, J. Endres, M. Wurm: "First steps towards a scatterometry reference standard", Proc. SPIE 8466 (2012) 84660E-1-8

[8] J. Endres, S. Burger, M. Wurm, B. Bodermann: "Numerical investigations of the influence of different commonly applied approximations in scatterometry" Proc. SPIE 8789 (2013) 8789-03 
[9] J. Endres, A. Diener, M. Wurm, B. Bodermann: "Investigations of the influence of common approximations in scatterometry for dimensional nanometrology" Meas. Sci. Technol. 25 (2014) 044004 (7pp)

[10] NIST SRM2059: https://www-s.nist.gov/srmors/view_detail.cfm?srm=2059

[11] J. Richter et al.: "Calibration of CD mask standards for the $65 \mathrm{~nm}$ node: CoG and MoSi", Proc SPIE 6533, 6533-53

[12] http://www.vlsistandards.com/products/dimensional/nanocd.asp?sid=83

[13] http://www.supracon.com/en/nanoscale_calibration.html

[14] http://www.nist.gov/manuscript-publication-search.cfm?pub_id=914808

[15] http://www.ptb.de/emrp/ind17.html, http://www.euramet.org

[16]R. M. Silver, N. F. Zhang, B. M. Branes, J. Qin, H. Zhou, R. Dixon: "A Baysian statistical model for hybrid metrology to improve measurement accuracy", Proc. SPIE 8083 (2012) 8083-07

[17] M.-A. Henn: "Statistical approaches to the inverse problem of scatterometry" PhD Thesis. (2013)

[18] S. Heidenreich, H. Gross, M.-A. Henn, C. Elster, M. Bär: "A surrogate model enables a Bayesian approach to the inverse problem of scatterometry" J. Phys.: Conf. Ser. 490, (2014) 1, 012007.

[19]Dai G. et al. Measurements of CD and sidewall profile of EUV photomask structures using CD-AFM and tiltingAFM, Meas. Sci. Technol. 25 (2014) 044002

[20] C. G. Frase ; D. Gnieser ; K. Dirscherl ; E. Buhr and H. Bosse: "A new SEM CD operator verified against Monte Carlo simulations", Proc. SPIE 6518 (2007), 65184P

[21] Victor Soltwisch; Jan Wernecke; Anton Haase; Jürgen Probst; Max Schoengen; Michael Krumrey; Frank Scholze; Jan Pomplun; Sven Burger: „Determination of line profiles on nano-structured surfaces using EUV and x-ray scattering“, Proc. SPIE 9235 (2014), 92351D

[22] Sven Burger; Lin Zschiedrich; Jan Pomplun; Frank Schmidt; Bernd Bodermann: „Fast simulation method for parameter reconstruction in optical metrology", Proc. SPIE 8681 (2013), 868119

[23] F. Scholze, B. Bodermann, S. Burger, J. Endres, A. Haase, M. Krumrey, C. Laubis, V. Soltwisch, A. Ullrich, J. Wernecke: "Determination of line profiles on photomasks using DUV, EUV, and x-ray scattering", Proc. SPIE 9231 (2014), 92310M

[24] Poul-Erik Hansen, Lars Nielsen: Setting up a metrological traceable Mueller polarimeter", presentation at the 8th Workshop Ellipsometry, 10- 12 March 2014, Dresden, Germany http://www.ipfdd.de/fileadmin/user_upload/ax/OEA/8th_Workshop_Ellipsometry/Book\%20of\%20abstracts.pdf

[25] M. Wurm, S. Bonifer, B. Bodermann, J. Richter: "Deep ultraviolet scatterometer for dimensional characterization of nanostructures: system improvements and test measurements", Meas. Sci. Technol. 22 (2011), 094024-1 - 094024-9

[26] J. Endres, M. Wurm, J. Probst, M. Schoengen, A. Diener, B. Bodermann: Determination of subwavelength grating structure geometry by optical scatterometry, submitted to Opt. Lett.

[27] V. Soltwisch, J. Wernecke, A. Haase, J. Probst, M. Schoengen, M. Krumrey, F. Scholze, "Nanometrology on gratings with GISAXS: FEM reconstruction and Fourier analysis,“ Proc. SPIE 9050, 905012 (2014) 